\title{
HIPONATREMIA INDUCIDA POR CICLOFOSFAMIDA EN ALTAS DOSIS: ESTUDIO DE COHORTE RETROSPECTIVA
}

Hyponatremia induced by high-dose cyclophosphamide therapy: a retrospective cohort study Cyclophosphamide and Hyponatremia

\author{
Bonella M. Belén ${ }^{1 \dagger}$, Warley Fernando ${ }^{1 \dagger}$, Gutierrez G Paula ${ }^{1}$, Elizondo M. Cristina ${ }^{2}$, Angriman Federico ${ }^{3}$, \\ Otero Victori ${ }^{4}$ y Ferreyro L. Bruno ${ }^{3}$
}

\section{Resumen:}

La hiponatremia asociada al uso de Ciclofosfamida es un efecto adverso infrecuente. Los estudios publicados no son concluyentes, especialmente respecto de los pacientes sometidos a dosis altas de dicho fármaco.

Objetivos: Determinar la incidencia de hiponatremia en una subpoblación de pacientes que realizaron tratamiento con dosis altas de Ciclofosfamida.

Materiales y Métodos: Estudio de cohorte retrospectivo de pacientes adultos que recibieron Ciclofosfamida a altas dosis en el período 20102014 en el Hospital Italiano de Buenos Aires. El evento primario fue la hiponatremia (definida como sodio plasmático menor a $135 \mathrm{meq} / \mathrm{L})$ mientras que como eventos secundarios se tomaron la hiponatremia sintomática y la hiponatremia severa (valor menor $120 \mathrm{meq} / \mathrm{L}$ ).

Resultados: 96 pacientes recibieron altas dosis de Ciclofosfamida durante el período estudiado. Se excluyó un $27.4 \%$ de los pacientes por cumplir con los criterios de exclusión y se analizaron los datos de 69 pacientes. La edad media de la población fue de 56.5 años (DS 12.8) y un $37.7 \%$ de los pacientes eran mujeres. La incidencia de hiponatremia fue de 52\% (IC95\% 39-64), hiponatremia severa $5.8 \% \quad(I C 95 \% \quad 0-12)$ y sintomática $8.7 \% \quad(I C 95 \%$ 1.3-16). La única variable asociada independientemente al desarrollo de hiponatremia fue el sexo femenino (OR: 3.89, IC95\% 1.02-8.55, p=0.04).

Si bien la incidencia observada resulta mayor a la observada en reportes previos, probablemente se deba a que solo consideramos la infusión de Ciclofosfamida a altas dosis. La hiponatremia severa y sintomática fue menor, aunque un $20 \%$ de los pacientes debieron prolongar su internación por esta causa.

Palabras clave: hiponatremia; ciclofosfamida; movilización stem cells hematopoyéticas, efecto adverso drogas

\begin{abstract}
:
Cyclophosphamide-associated hyponatremia is an uncommon adverse effect. Published reports are inconclusive regarding patients under high-dose therapy.

Objective: To determine the incidence of hyponatremia in patients under high-dose cyclophosphamide therapy.

Methods: Retrospective cohort of adult patients who received high-dose cyclophosphamide therapy between 2010 and 2014 at the Hospital Italiano de Buenos Aires. The primary end-point was hyponatremia (defined as plasma sodium levels $<135$ $\mathrm{mEq} / \mathrm{L}$ ), and secondary end-points were symptomatic hyponatremia, severe hyponatremia (plasma sodium levels $<120 \mathrm{mEq} / \mathrm{L})$.

Results: A total of 96 patients received high-dose cyclophosphamide therapy during the study period. 27 patients met exclusion criteria and accounted for $28.1 \%$, so that 69 patients were included in the primary analysis. Study population mean age was 56.5 years (SD 12.8) and $37.6 \%$ were female. The cumulative incidence were as follows: $52 \%(\mathrm{Cl} 95 \%$ 39-64) for hiponatremia, 5.8\% (Cl 95\% 0-12) for severe hyponatremia, and $8.7 \%(\mathrm{Cl} 95 \% 1.3-16)$ for symptomatic hyponatremia. The only independent variable associated with the development of hyponatremia was female gender (OR 3.89, CI 95\% 1.02-8.55, $\mathrm{p}=0.04$ ).

Cumulative incidence found in this study appears higher than in previous reports, probably because only patients under high-dose cyclophosphamide therapy were included. Cumulative incidence of severe and symptomatic hyponatremia were lower
\end{abstract}

Key words: hyponatremia; cyclophosphamide; hematopoietic stem cell mobilization; drug side effects. 


\section{Introducción}

La Ciclofosfamida es un agente alquilante utilizado en múltiples esquemas terapéuticos para el tratamiento de enfermedades oncológicas y reumatológicas. Este fármaco puede utilizarse a dosis bajas, menores a 30 $\mathrm{mg} / \mathrm{kg} / \mathrm{día}$, o a dosis altas, mayores a 40 $\mathrm{mg} / \mathrm{kg} /$ día1,2. Esta última es utilizada en pacientes que realizan movilización de stem cells hematopoyéticas (MSCH) como preparación para el trasplante de médula ósea, en el tratamiento de ciertos tipos de linfoma y en el tratamiento de pacientes con leucemia linfoblástica aguda con esquema GATLA o como parte del esquema HyperCVAD.2

Los efectos adversos comúnmente descritos incluyen cistitis hemorrágica, mielosupresión, alopecia, hiperpigmentación de las uñas y esterilidad. La toxicidad limitante suelen ser los efectos adversos hematológicos2. Dentro de las reacciones infrecuentes se incluye la hiponatremia. Con respecto a la misma, se proponen dos mecanismos posibles relacionados a su desarrollo: un mecanismo de tipo síndrome de secreción inadecuada de hormona antidiurética por estímulo directo a nivel hipofisario y un segundo mecanismo a nivel renal donde la Ciclofosfamida competiría con la ADH aumentando la permeabilidad al agua en el túbulo distal 1,2,5,6. Los estudios no son concluyentes con respecto a la frecuencia de este efecto adverso y actualmente sólo disponemos de series de casos 0 estudios retrospectivos $y$ prospectivos en pequeña escala con dosis bajas de Ciclofosfamida 3,7-9. La incidencia de hiponatremia en estos trabajos ronda del $10 \%$ al $25 \%$ pero no hay datos acerca de los pacientes sometidos a dosis altas del fármaco 1,9 .

Diseñamos un estudio de cohorte retrospectivo para analizar una subpoblación de pacientes sometidos a tratamiento con Ciclofosfamida a alta dosis con el objetivo de establecer la incidencia de hiponatremia. Nuestro objetivo global es describir la magnitud del problema y detectar factores de riesgo asociados al desarrollo de hiponatremia en pacientes bajo tratamiento con Ciclofosfamida. Las implicancias de estos resultados podrían contribuir a una mejor prevención y manejo de esta toxicidad.

\section{Material y Métodos}

\section{Diseño}

Se realizó un estudio de cohorte retrospectivo de pacientes adultos que recibieron Ciclofosfamida a altas dosis en el período 2010 a 2014 en el Hospital Italiano de Buenos Aires, Argentina, centro de tercer nivel universitario. El presente estudio fue realizado de acuerdo con la declaración de Helsinki y reportado según las guías STROBE 10.

\section{Participantes}

Se realizó un muestreo no aleatorio y de tipo consecutivo de aquellos pacientes que acudieron al servicio de Hematología y Oncología del Hospital Italiano de Buenos Aires para recibir tratamiento con Ciclofosfamida a altas dosis (mayor a 2.5 gramos). Los datos fueron extraídos de los registros de la farmacia central del Hospital. En nuestro centro, esta población está compuesta principalmente por aquellos pacientes que realizan $\mathrm{MSCH}$ y un subgrupo de pacientes que reciben los esquemas HyperCVAD y GATLA (dado que en estos casos la dosis se ajusta a la superficie corporal). Se registraron únicamente aquellos pacientes que realizaron un primer ciclo de HyperCVAD/GATLA. Los pacientes que no presentaban un registro de sodio previo o posterior a la infusión de Ciclofosfamida fueron excluidos del análisis primario.

\section{Mediciones y variables explanatorias}

Los datos demográficos y las características clínicas de los participantes fueron recabados de una base de datos electrónica secundaria. Se registraron de cada paciente variables demográficas, clínicas y de laboratorio. Los datos fueron recabados por dos investigadores principales en una base de datos electrónica. Las variables explanatorias $y$ el evento de interés fueron recabados por investigadores independientes a fin de evitar el sesgo de información potencial. Finalmente, el coordinador de la investigación se encargó de conciliar los datos a fin de encontrar valores erróneos y datos faltantes. Específicamente se registraron: edad, sexo, diagnóstico basal, dosis administrada, comorbilidades y medicación concurrente. Asimismo, se recabaron los valores de sodio 
previo y posterior a la infusión de Ciclofosfamida. En nuestra institución, los pacientes que realizan $\mathrm{MSCH}$ rutinariamente tienen un control de natremia posterior a la infusión y en algunos casos al día siguiente. En cambio, en los pacientes que realizan el esquema HyperCVAD o GATLA el control de sodio queda a criterio del médico tratante.

\section{Eventos}

Se definió como evento primario la ocurrencia de hiponatremia (sodio plasmático menor a $135 \mathrm{meq} / \mathrm{L})$ posterior a la infusión. Como eventos secundarios se definieron la ocurrencia de hiponatremia severa (valor menor $120 \mathrm{meq} / \mathrm{L})$, la hiponatremia sintomática, la magnitud del descenso de los valores de sodio y la prolongación de la internación por esta causa. Se definió hiponatremia clínicamente significativa a la ocurrencia de hiponatremia severa y/o sintomática considerando que la misma es la que en la práctica lleva a una conducta activa para corregir el descenso del sodio. Para todas estas variables se consideró un tiempo al evento de hasta 7 días posteriores a la infusión de Ciclofosfamida.

\section{Análisis estadístico}

Las variables cuantitativas se reportan como media, desvío standard o mediana e intervalo intercuartil según la distribución observada, y las variables categóricas se reportan como proporciones. Se realizó un análisis bivariado comparando las características basales y clínicas entre los pacientes que desarrollaron o no el evento primario. La comparación de variables continuas se realizó con test de Student o Wilcoxon según distribución. La comparación de variables categóricas se realizó con el test de Chi Cuadrado. Para identificar variables asociadas al desarrollo de hiponatremia en contexto de otras variables, se realizó un análisis multivariado utilizando un modelo de regresión logística. Se incluyeron en el análisis aquellas variables consideradas clínicamente relevantes a priori así como variables significativas en el análisis bivariado. Se consideraron significativas las $p$ menores a 0.05 . El tamaño muestral fijo se alcanzó con los pacientes que recibieron altas dosis de Ciclofosfamida en el período estudiado.
Al ser esperable una importante proporción de pacientes sin controles de sodio que serían excluidos del análisis primario, se realizaron diversos análisis de sensibilidad simulando distintos escenarios. En primer lugar, como muchos de los pacientes excluidos no tenían controles de sodio previo, pero si posteriores fue posible estimar la incidencia de hiponatremia en este subgrupo comparándola con la población analizada. En segundo lugar, se estimó la incidencia global de hiponatremia en el escenario que ninguno de los pacientes excluidos haya desarrollado el evento.

Se utilizó el software SPSS v.19 para todos los análisis.

\section{Resultados}

\section{Participantes}

Entre 2010 y 2014 recibieron Ciclofosfamida a altas dosis 96 pacientes. 27 pacientes $(28.1 \%)$ fueron excluidos del análisis primario. El principal motivo para la exclusión de pacientes fue que no presentaban mediciones de sodio basales por lo cual no podría adjudicarse el evento como nuevo, también se excluyeron los pacientes que no tenían controles de sodio posterior a la infusión de Ciclofosfamida. Finalmente, se analizaron los datos de 69 pacientes (Figura 1).

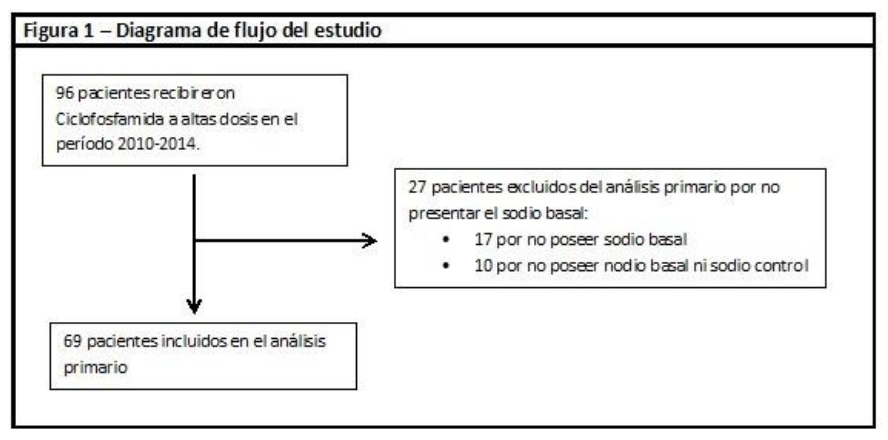

En cuanto a las características basales, la edad media de la población fue de 56,5 años (DS 12.8) y un $37.7 \%$ fueron mujeres (Tabla 1). 
Tabla 1 - Car acter ísticas basales de los pacientes incluidos en el análisis primario con Ciclofosfamida a altas dosis.

\begin{tabular}{lrr}
\hline & $\mathrm{N}=69$ \\
\hline Factores demográficos & Edad, media (DS) & $56.5(12.8)$ \\
& Mujeres N (\%) & $26(37.7)$
\end{tabular}

Hábitos N\%

Tabaquismo $\quad 4(5.8)$

Alcoholismo $1(1.4)$

Condicionesclínicas $\mathrm{N} \%$

$\begin{array}{rc}\text { Insuficiencia cardiaca } & 2(2.9) \\ \text { Insuficiencia renal } & 9(13.0) \\ \text { Enfermedad hepática } & 1(1.4) \\ \text { Hipotiroidismo } & 5(5.2) \\ \text { Diabetes mellitus } & 5(7.2) \\ \text { EPOC } & 1(1.4) \\ \text { Accidentecerebrovascular } & 2(2.9) \\ \text { Hiponatremia previa } & 1(1.4)\end{array}$

Fármacos $\mathrm{N} \%$

$\begin{array}{rc}\text { Psicofármacos } & 26(37.7) \\ \text { Diuréticos } & 1(1.4) \\ \text { IBP } & 18(26.0) \\ \text { AlNES } & 9(13.0) \\ \text { Opioides } & 12(17.4) \\ \text { Corticoides } & 26(37.7) \\ \text { Antibióticos } & 16(23.0) \\ \text { Quimioterapia reciente } & 32(46.4) \\ \text { Ciclofosfamida baja dosis reciente } & 11(15.9)\end{array}$

Esquemas quimioterápicos $\mathrm{N} \%$

$\begin{array}{rr}\text { Movilización } & 54(78.3) \\ \text { HyperCVAD } & 10(14.5) \\ \text { GATLA } & 5(7.2)\end{array}$

Eventos \% (IC 95\%)
$52(39-64)$

$5,79(0-12)$

$8.7(1.3-16)$

$20(10-30)$

Abreviaturas DS: Desvio standard, $N$ \%: número y porcentaje, EPOC enfermedad pulmona obstructiva crónica, IBP: Inh bidor de la bomba de protones, AINES: antiinflamatorios no esteroides, HyperCVAD Ciclofosamida, Vincristina, Adriamicina y Dexametasona, GATLA Ciclofosamida, 6 Mercaptopurina, Citosina Arabinósido, terapia intratecal con Metr otexate, IC95\%: Intervalo de Confianza $95 \%$.

La población analizada presentaba un sodio basal de 137 (DS 2.86) y un 78,3\% realizaron $\mathrm{MSCH}$. Un 46,4\% realizó quimioterapia previamente, siendo Ciclofosfamida a bajas dosis un $15.9 \%$. En cuanto a las condiciones clínicas asociadas las más frecuentes fueron: insuficiencia renal $(13 \%)$, hipotiroidismo $(5.2 \%)$ y diabetes mellitus $(7.2 \%)$. Los fármacos más frecuentemente utilizados como medicación habitual de los pacientes previo y durante la infusión de Ciclofosfamida fueron psicofármacos y corticoides $(37.7 \%$ en ambos casos).

\section{Eventos}

La incidencia de hiponatremia posterior a la infusión de Ciclofosfamida a altas dosis fue del $52 \%$ (IC95\% 39-64). Un 5,8\% (IC95\% 0-12) de los pacientes presentó hiponatremia severa y un 8,7\% (IC95\% 1.3-16) hiponatremia sintomática, siendo el evento más grave reportado la incidencia de convulsiones que se registraron en un sólo paciente. En los pacientes que desarrollaron hiponatremia, la media de descenso de sodio fue de $6.7 \mathrm{meq} / \mathrm{L}$ (DS 6,21). La media del tiempo desde la infusión de Ciclofosfamida hasta el desarrollo del peor valor de sodio fue de 35 hs (DS 25,7) y un 20\% (IC95\% 10-30) debieron prolongar su internación por hiponatremia. La incidencia de hiponatremia clínicamente significativa fue de 10.1\% (IC95\% 216).

En segunda instancia, se compararon la distribución de las diferentes covariables basales entre los pacientes que desarrollaron y los que no desarrollaron hiponatremia. En el análisis bivariado, la única covariable asociada con el desarrollo de hiponatremia fue el sexo femenino ( $50 \%$ vs $24.2 \%, p=0.03$ ). No se encontraron diferencias significativas en las variables demográficas, la carga de comorbilidad o el uso de fármacos entre ambos grupos (Tabla 2).

\begin{tabular}{|c|c|c|c|}
\hline & $\begin{array}{l}\text { Hiponatremia } \\
(\mathrm{N}=36)\end{array}$ & $\begin{array}{c}\text { Sin hiponatremia } \\
(N=33)\end{array}$ & Valor $\mathrm{p}^{1}$ \\
\hline \multicolumn{4}{|l|}{ Factores demográficos } \\
\hline Edad, media (DS) & $58.3(11.2)$ & $54.5(14.3)$ & 0.22 \\
\hline Mujeres N (\%) & $18(50.0)$ & $8(24.2)$ & 0.03 \\
\hline \multicolumn{4}{|l|}{ Hábitos N\% } \\
\hline Tabaquismo & $2(5.5)$ & $2(6.1)$ & 0.92 \\
\hline Akoholismo & 0 & $1(3.0)$ & 0.36 \\
\hline \multicolumn{4}{|l|}{ Condiciones clínicas $\mathrm{N} \%$} \\
\hline Insuficiencia cardiaca & 0 & $2(6.1)$ & 0.83 \\
\hline Insuficiencia renal & $9(25.0)$ & $3(9.1)$ & 0.80 \\
\hline Enfermedad hepática & $1(28)$ & 0 & 0.53 \\
\hline Hipotiroidsmo & $5(13.9)$ & $1(3.0)$ & 0.52 \\
\hline Diaberes mellitus & $5(13.9)$ & $3(9.1)$ & 0.54 \\
\hline EPOC & $1(2.8)$ & 0 & 0.47 \\
\hline Accidente cerebrovæcular & $2(5.5)$ & $1(3.0)$ & 0.37 \\
\hline Hiponatremia previa & 0 & $1(3.0)$ & 0.25 \\
\hline \multicolumn{4}{|l|}{ Fármacos $(\mathrm{N} \%)$} \\
\hline Psicofár macos & $10(27.8)$ & $10(30.3)$ & 0.81 \\
\hline IBP & $6(16.7)$ & $12(36.4)$ & 0.06 \\
\hline AINES & $3(8.3)$ & $6(18.2)$ & 0.22 \\
\hline Opioides & $7(19.4)$ & $5(15.2)$ & 0.63 \\
\hline Corticoides & $12(33.3)$ & $14(42.4)$ & 0.43 \\
\hline Antbióticos & $8(22.2)$ & $8(24.2)$ & 0.84 \\
\hline Quimioterapia reciente & $17(47.2)$ & $15(45.5)$ & 0.88 \\
\hline Ciclofosfamida baja dosis reciente & $7(19.4)$ & $4(12.1)$ & 0.40 \\
\hline \multicolumn{4}{|l|}{ Esquemas quimioterápicos ( $\mathrm{N} \%$ ) } \\
\hline Movilización & $31(86.1)$ & $23(69.7)$ & 0.10 \\
\hline HyperCVAD & $4(11.1)$ & $6(18.2)$ & 0.10 \\
\hline GATLA & $1(28)$ & $4(12.1)$ & 0.10 \\
\hline
\end{tabular}

${ }^{1} \mathrm{P}$ valor calculado utilizando Test de Student o Wiicoxon para var iables continuas según distr bución y Chicuadrado para variables caegóricas.

Abreviaturas DS: Desvio standard, $N \%$ : núme o y porcentaje, EPOC: enfermedad pulmonar obstructiva crónica, IBP: Inhibidor de la bomba de protones AINES: antinflamatorios no ester oides HyperCVAD: Ciclofosamida, Vincristina, Adriamicina y Dexametasona, GATLA: Ciclofosfamida, 6 Mercaptopurina, Citosina Arabinósido, terapia intratecal con Metrotexae. 
Es de notar que un $86.1 \%$ de los pacientes realizó $\mathrm{MSCH}$ en el grupo hiponatremia vs $69.7 \%$ del grupo no hiponatremia.

Finalmente, se utilizó un modelo de regresión logística para evaluar la asociación entre las distintas covariables y el desarrollo de hiponatremia. El sexo femenino fue la única covariable asociada al desarrollo de hiponatremia (OR 3.89 IC95\% 1.02-8.55, p=0.04). En el modelo multivariado que incluyó a las variables sexo, edad, $\mathrm{MSCH}$ y enfermedad que predisponga a hiponatremia (definida como la combinación de insuficiencia cardiaca, insuficiencia renal y enfermedad hepática), el sexo femenino mantuvo su efecto, observándose que el ser mujer aumenta el riesgo de desarrollo de hiponatremia (OR 1.08, IC95\% 1.02-8.55, $\mathrm{p}=0.05$ ) (Tabla 3).

abla 3 - Odds Radio (OR) e intervalo confianza 95\% (IC95\%) parael desarrollo de hiponatremia según covariables asales

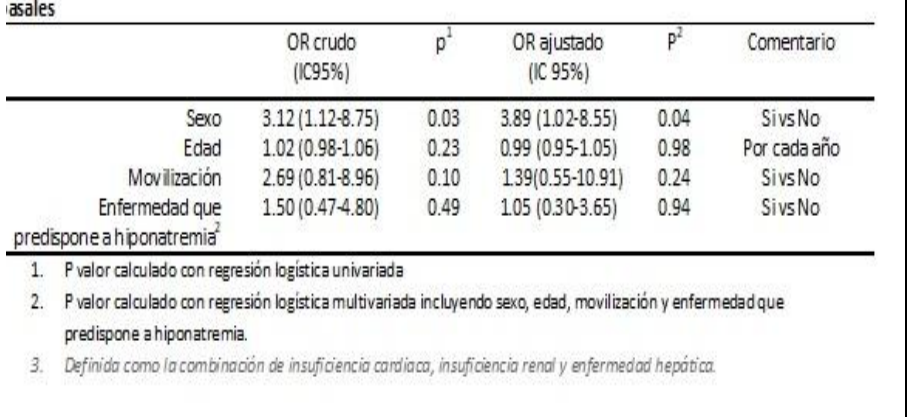

\section{Análisis de sensibilidad}

Se realizó el análisis de sensibilidad preestablecido con los 27 pacientes excluidos, encontrándose una incidencia de hiponatremia del 33.3\% (IC95\% 13-52) (Apendice 1). La incidencia de hiponatremia incluyendo todos los pacientes que tuvieron control de sodio posterior fue del $46.9 \%$ (IC95\% 36-57), mientras que la incidencia global de hiponatremia en el escenario que ninguno de los pacientes excluidos haya desarrollado el evento fue de $37 \%$ (IC95\% 2747). Todos los pacientes excluidos pertenecían al grupo $\mathrm{MSCH}$. No se registraron casos de hiponatremia severa o sintomática y sólo un paciente debió prolongar su internación por esta causa. Al evaluarse las condiciones clínicas asociadas se encontró que las poblaciones eran similares mientras que hubo diferencia en el consumo de fármacos: psicofármacos (3.7\%), IBP (0\%) y antibióticos (3.7\%), vs $37.7 \%, 26 \%$ y $23 \%$ respectivamente.

\section{Discusión}

Nuestro estudio describe la incidencia de hiponatremia en una población expuesta a Ciclofosfamida a altas dosis en un hospital universitario de tercer nivel. La incidencia estimada fue de un $52 \%$ y el promedio del descenso de sodio de $6.7 \mathrm{meq} / \mathrm{L}$. Sin embargo, la incidencia de hiponatremia sintomática y severa fue menor (5.8\% y $8.7 \%$ respectivamente). Si bien la implicancia clínica del evento puede haber sido escasa en muchos pacientes, un 10.1\% presentó hiponatremia clínicamente significativa, lo que implica en la práctica una conducta activa para su corrección. Más aún, un $20 \%$ de los pacientes debió prolongar su internación por dicha situación clínica. La única variable que mostró una asociación significativa en el modelo multivariado fue el sexo femenino.

La incidencia de hiponatremia en nuestra población ha sido considerablemente superior que estudios previos, donde se reportan cifras de un $10 \%$ a un $25 \% 1$. Esta diferencia puede explicarse por el hecho de que nuestro estudio se realizó únicamente con pacientes que recibieron Ciclofosfamida a altas dosis. Creemos que la baja frecuencia de hiponatremia severa 0 sintomática puede deberse a la detección precoz y rápida intervención médica para corregir la caída del sodio, sumado a que los pacientes que realizan estos tratamientos reciben medicación antiemética, analgésicos y corticoides que podrían atenuar los síntomas y de esta forma disminuir concomitantemente la adjudicación de síntomas a la hiponatremia en sí. Consistente con la literatura, los pacientes del presente estudio presentan una baja frecuencia de comorbilidades; esto podría deberse a que en su mayoría son pacientes que realizaron $\mathrm{MSCH}$ y se encuentra en plan de trasplante de Médula Ósea. De manera interesante, si bien estudios previos han reportado la asociación entre el sexo masculino y el desarrollo de hiponatremia, en nuestro análisis multivariado se muestra una asociación inversa3. Esto es consistente con otros estudios en contextos clínicos disímiles que evidenciaron que el sexo femenino es un factor de riesgo independiente para el desarrollo de hiponatremia inducida por drogas 11.

El presente estudio presenta potenciales limitaciones. En primer lugar, un porcentaje considerable de nuestra población inicial tuvo que ser excluida del análisis primario por no contar con valores de sodio basales, pudiendo sobreestimar la incidencia del evento de interés. Sin embargo, un análisis de sensibilidad 


\section{Hiponatremia inducida por Ciclofosfamida en altas dosis: estudio de cohorte retrospectiva}

conservador en donde se consideró que ningún paciente excluido presentó hiponatremia mostró una incidencia de $37 \%$ fortaleciendo la robustez de nuestros hallazgos. En segundo lugar, los pacientes incluidos podrían presentar características suficientemente distintas a los excluidos, constituyendo una población no representativa. Sin embargo, un análisis comparativo entre ambas poblaciones no mostró diferencias significativas (ver apéndice suplementarios). En tercer lugar, los datos fueron relevados de bases secundarias por lo que se debe considerar el riesgo del subdiagnóstico de los síntomas de hiponatremia, las condiciones clínicas asociadas, el consumo de fármacos y el antecedente de hiponatremia previa. Creemos que esta limitación subestimaría la incidencia de hiponatremia clínicamente significativa, observada en hasta un $10 \%$ de nuestra población. En cuarto lugar e inherente al tipo de diseño, existen un gran número de variables asociadas al desarrollo de hiponatremia que no pudimos evaluar adecuadamente, tales como el índice de masa corporal y el consumo de medicación concomitante. Por último, si bien la incidencia de hiponatremia observada es elevada, este estudio carece de información que nos permita seleccionar mejor aquella población con riesgo de tener eventos clínicamente significativos.

En contrapartida, nuestro estudio presenta varias fortalezas. En primera instancia, es el primer estudio que analiza una cohorte numerosa de pacientes sometidos a Ciclofosfamida a altas dosis. En segundo lugar, la elevada incidencia de hiponatremia observada fortalece la conducta del control estricto de laboratorio que debe realizarse en estos pacientes. En tercer lugar, fue posible estimar las cifras de hiponatremia clínicamente significativa, estimando que 1 de cada 10 pacientes presentaron un evento que requiere una conducta médica activa. Más aún, como se explicó anteriormente, es probable que esta cifra haya sido subestimada por cuestiones inherentes al diseño. En cuarto lugar, estos hallazgos actúan como disparadores de hipótesis para futuros estudios que intenten detectar factores de riesgo para el desarrollo de hiponatremia, como el sexo femenino. Por último, los hallazgos podrían ser de interés para las autoridades dado que la estancia hospitalaria podría prolongarse asociada a la hiponatremia.

En conclusión, considerando los resultados obtenidos, se fortalece la recomendación del control clínico y de laboratorio de todos los pacientes que realizan Ciclofosfamida a altas dosis. Si bien los pacientes que se someten a trasplante de médula ósea constituyen una población heterogénea, comparten ciertas características comunes que hacen estos hallazgos potencialmente generalizables a otros centros. Esto podría tener implicancia para un mejor cuidado y seguimiento de estos pacientes. De todas formas, consideramos que los hallazgos deben ser reproducidos en estudios prospectivos.

\section{Bibliografía}

1. Lee YC, Park JS, Lee CH, Bae SC, Kim IS, Kang CM, et al (2010) Hyponatraemia induced by low-dose intravenous pulse cyclophosphamide. Nephrology, dialysis, transplantation : official publication of the European Dialysis and Transplant Association - European Renal Association 25(5):1520.

2. Laurence B BC (2012) Goodman y Gilman's: The Pharmacological basis of therapeutics. McGraw $\mathrm{H}$, editor.

3. Koo TY, Bae SC, Park JS, Lee CH, Park MH, Kang CM, et al (2007) Water intoxication following low-dose intravenous cyclophosphamide. Electrolyte \& blood pressure : E \& BP 5(1):50-54.

4. Lazarevic V, Hagg E, Wahlin A (2007) Hiccups and severe hyponatremia associated with high-dose cyclophosphamide in conditioning regimen for allogeneic stem cell transplantation. American journal of hematology 82(1):88.

5. Gilbar PJ, Richmond J, Wood J, Sullivan A (2012) Syndrome of inappropriate antidiuretic hormone secretion induced by a single dose of oral cyclophosphamide. The Annals of pharmacotherapy 46(9):e23.

6. Bruining DM, van Roon EN, de Graaf $H$, Hoogendoorn $M$ (2011) Cyclophosphamide-induced symptomatic hyponatraemia. The Netherlands journal of medicine 69(4):192-5.

7. Harlow PJ, DeClerck YA, Shore NA, Ortega JA, Carranza $A$, Heuser $E$ (1979) $A$ fatal case of inappropriate $A D H$ secretion induced by cyclophosphamide therapy. Cancer 44(3):896-8.

8. Park SJ, Kim JH, Shin Jl (2010) Insight on mechanism of hyponatraemia induced by low-dose intravenous pulse cyclophosphamide. Nephrology, dialysis, transplantation : official publication of the European Dialysis and Transplant Association - European Renal Association 25(10):3453; author reply -4

9. Alilou M, Awab A, Zarouf M, Moussaoui RE, Hijri AE, Azzouzi A, et al (2009) Severe hyponatraemia secondary to cure of cyclophosphamide (about three cases). Annales francaises d'anesthesie et de reanimation 28(1):103-4.

10. Sharp S PM, Thompson S, White I, Wood A (2014) A review of published analyses of case-cohort studies and recommendations for future reporting. Plos One. 9(6):1-6.

11. Chow KM, Szeto CC, Wong TY, Leung CB, Li PK (2003) Risk factors for thiazide-induced hyponatraemia. QJM : monthly journal of the Association of Physicians. 96(12):911-7. 